\title{
STUDY OF DEFECTS, TESTCASES AND TESTING CHALLENGES IN WEBSITE PROJECTS USING MANUAL AND AUTOMATED TECHNIQUES
}

\author{
Bharti Bhattad ${ }^{1}$ and Dr. Abhay Kothari ${ }^{2}$ \\ ${ }^{1}$ M.tech in Software Engineering under guidance of Abhay Kothari \\ bhartibhattad118@gmail.com \\ ${ }^{2}$ Department of Computer Engineering
}

\begin{abstract}
Testing is the one of the important component of any software engineering process. As we talking about the software's applications then web application is the fastest growing application now a day. So web application or web sites will be tested accurately and correctly. Web testing includes testing of various applications like configuration control, navigation control, state, database etc. Web site testing ensures that there will be no broken links, no images will be missed, there should be no spelling mistakes, no any errors or bugs in software, and the download time should not be so delay as specified. Timeliness, structural quality, content, accuracy and consistency, response time and latency and performance are the major web site's quality factors. Functional, browser, performance, security, usability, database etc testing are performed on any website to make it defect free. Also for any project we also need to maintain the database. So database plays very important role for every organization, so for better results testing of database is required. It is now not only the necessity of project or web application itself but of the organization also to avoid any future problems that can be come in application. As a minute fault in data base can causes data loss that may be uncover able in future. Many tools and frameworks are available for testing of databases or generate test cases to check the applications. When we test the website or any web application and there is difference between expected results and the actual results, there is defect. Defects can be classified in to 3 categories: Wrong, Missing and Extra. Errors can be classified according to priority or severity. According to the severity and priority of the defects, these can be fixed before deliver product to the client. In this paper we represent that on which we can apply tests in on database how we can perform testing on database. We have also computed the coverage of design of test cases to maintain the quality of testing. By this, we can decrease the time, memory and cost of project to some extent, there by easing the tester to manage their testing phases easily.
\end{abstract}

\section{KEYWORDS}

Manual/Automation Testing, Defects, Defect types, Test cases design, Database testing

\section{INTRODUCTION}

Testing is very important for any software which is developed by any organization. This is one of the major activities of any software development life cycle as software consists of number of program to perform some different or specific tasks. Software Testing ensures that software which will be deployed to any client it will be error free .Testing is used to demonstrate the David C. Wyld et al. (Eds) : COSIT, DMIN, SIGL, CYBI, NMCT, AIAPP - 2014 pp. 79-89, 2014. @ CS \& IT-CSCP 2014

DOI : $10.5121 /$ csit.2014.4907 
validity of software at each and every stage. As software testing directly affects the quality of software. Generally large organizations spend $60 \%$ of their development cost on testing [11]. Software testing is the phase in which testers identify defects, defect is any variance between actual and expected output. Software testing is the phase for analyzing the software for the purpose of identifies the errors so that the software become error free and clients not get any defects at their own side. This directly deals with the quality of software. Database is one of the important entities of any project also database applications are very complex to handle as it consists of Many components and layered architecture. Testing of database is very essential that database should accept correct data or it perform functions correctly and it must perform acceptable performance when deployed .In this paper we include necessity of software with its types, web site quality, test cases design, and types of testing for web site, defect and its life cycle, database testing, and at last it concluded with when to stop testing and also which defect has been removed on the basis of priority and severity of defects. In this we include the importance of database testing. There may be number of approaches are there to test the database application but currently we discussing about two approaches. With the first approach, application developers carry out their tests for databases on their local database. But this approach cannot fulfill the needs of all the testing stages, especially performance and scalability, due to the limitation of small size of data and test cases. Further more data in local development databases may not be accurate or close to real data as all data will create by developers so they know all valid and invalid conditions but data will be tested on user's point of view. Approach, applications are tested over live databases. But this approach can't be used always as security point of view like high risk of secure data disclosing [11]. Testing of database is different from testing of structural programs as access of database takes through SQL statements so it's important to do right testing for database and rest of codes. A database involves inputs like user's side input is given and instances of databases. In addition to check the expected output with original output some following things has to be checked like:

\section{Consistency of database.}

2. Reflect database to original environments [11].

Normally in database testing, as in theory a test run does not fail, if all request and responses are right and database states correct after execution of test run.

\subsection{Necessity of software testing:}

To find that that software is performing what it supposed to do and software not performing what it is not supposed to do. Basically software testing is doing to find the defects as soon as possible and get them fixed that means it's not all about to correct the code but to search the bugs or find the errors in code in starting phase and try to correct them in shortest possible time. If tester is not got any error then it indicates that testing process required improvement of test cases. So it's the necessity of testing process that the testers must write accurately test cases so that it can find out hidden defects from the programs.

\subsection{Types of software:}

Software development takes places so many types of applications are build up like desktop applications web applications etc. but web applications are the fastest growing application as we concern about the software application .various applications are coming in market or can be downloaded from play stores so these deploy over internet so that many users are get aware of these and can be use them easily so these must not contain any error. Therefore web application will be tested with maximum accuracy. So web testing includes various tests like load control, 
navigation control, configuration etc. web site testing says that there not be any link which is broken, there not be any missing data, values or images n, no spelling mistakes will be there, no bugs will be found etc must be taken in to account.

\subsection{Web site Quality:}

As there are many sites are launching day by day, and many application are performing or depending on the web sites so there must be maintain the quality of the website so that batter results can be found out. As there are many websites available user s will quickly move to different website if it is very slow or complex.

For example many e-commerce sites are running on different web sites and on that various transactions are performed on that so if there is any error on these sites then it directly affect the transactions operations, sales or any other major operation. So there must be some factors on the basis of that these web sites can be tested and quality can be maintained. Miller gives some quality factors like:

Timeliness: All the web pages of any web site must be upgraded time to time or daily according to the requirement.

\section{Structural quality:}

Content, accuracy, consistency, response time, latency and performance are important factors to maintain the structural quality.

\subsection{Software development life cycle models:}

The choice of specific development life cycle model depends on many things like availability of clear user requirements at the starting, whether the user is from IT background, whether there are major risks associated with the project, whether the product development includes high end research etc.

\begin{tabular}{|l|l|}
\hline $\begin{array}{l}\text { Development life cycle } \\
\text { model }\end{array}$ & Features \\
\hline Water fall model & $\begin{array}{l}\text { It uses when SRS document is clear. It is simple and } \\
\text { effective when requirements are clear. Monitoring of project } \\
\text { is simple as defined results or outputs are already available at } \\
\text { the end of each stage. }\end{array}$ \\
\hline Prototype model & $\begin{array}{l}\text { It uses when requirements are not clear. Prototyping is done } \\
\text { at developer's cost.eve }\end{array}$ \\
\hline $\begin{array}{l}\text { Evolutionary } \\
\text { development model }\end{array}$ & Similar to prototype model to some extent. \\
\hline Spiral model & $\begin{array}{l}\text { In this some financial conditions is to be checked again and } \\
\text { again. }\end{array}$ \\
\hline
\end{tabular}

\section{TeST CASES DESIGN}

For right testing process one need to take correct input and makes accurate test cases. Test cases are the set of expected inputs and expected outputs developed for particular objectives. According to IEEE STD 610 (1990) defines test case as set of inputs, execution conditions, and expected 
outputs developed for a particular objectives, such as to exercise a particular path or to verify compliance with a specific requirement.

Some important features that one should kept in mind:

- Test cases should covers all essential features be balanced. There is balance between all conditions like normal, abnormal and boundary conditions.

- All testing methods will be balanced whatever use like black box, white box, functional or non functional testing.

- Test cases must be accurate, economical repeatable, traceable, self understandable and self standing that means anyone who use the test cases are get the concepts means it should not be so complex.

- Test case must be according to need of description that to be tested that is it must be specific it should not contain any unspecific steps so unnecessary steps must be avoided.

- A good test case is one which give same result each time no maters that who is going to use it. A test case will be appropriate for developers, testers and environment.

Above are the necessity of good test cases but writing good test cases are the another major concept so for writing good test cases some of important things are:

- Improve testability of test cases: testability means easy to test. Test steps will be written in active cases so that all things will be clear and understandable to every person who will going to execute it.

- Improve accurately: accurately means testers follow the test directions, the result of test case pass or fail will be right. While designing the test cases some common mistakes has been done by testers that must be avoided like:

- Don't make too long test cases. Don't combine two or more test cases into one as a single test case may contain many results or verify many criteria.

- Incorrect, in appropriate test cases causes many confusions to testers or the person who going to test.

\section{TYPES OF TESTING FOR WEBSITE:}

Functional testing: In functional testing various functionalities of the web pages are get tested. It includes various check links, web forms, session testing, css tags, and dynamic contents testing for web pages. Check links includes test a link of a page to other external links, means all links given on the web page will be work properly and accurately. Testing of web forms include for the default values assigned on the web pages, password field for login purpose not show its content that it must be hide from other users, fake input values not be entered or taken for each and every field of the web page. Session testing shows that session cookies must be reset between browser session etc .in this various html ids, attribute all must be identified. It also includes database checking that is data consistency must be maintain, various create ,alter etc commands works properly, data should be give correct data on data retrieval option given by user. Data connectivity must be maintained throughout the session.

Browser compatibility testing: In browser testing various functions check like web application will work on several browsers or not that is it will be compatible to all the browsers equally and correctly, also check browser's security checking like hacking etc.web application must be compatible to operating environment that is check for user interface, desktop integration functionalities. 
Performance testing: In this web application can be checked for load and stress that is linear scalability it means web application's performance does not vary as the number of users increases. Load testing identifies scalability index for web application performance. Also check for response of server to browser when submitting request. Also check response of server over different time period. Stress testing specifies how application responding if level of load is very high.also checks the parts of web application if fails under heavy load. Also check for the functions that are responding if load is there and application get fails.

Security testing: As security is another major issue of the web application or any web site. This includes check for different URL's basic authentication should be check, check for invalid inputs and various text fields, check for web site protection of inaccessible web files or directories, check for insecure page for web sites etc should be checked.

Usability testing: This gives user interface with the website; it has large impact on the website. It includes test for navigation control that is page will be easily movable from one page to another, user find instructions for each functions that they require to operate, consistency must be maintain on each page that is from first to last, function should be work properly for each page. Apart from navigation it also includes content checking that is spelling errors must be avoided, content must be arranged logically and correctly, content must be easily understandable for the users, pattern style must be correct that is color, alignment, border, guidelines, frames, fonts etc must be checked.

Production monitoring: This check that web application must be test timely and save the service level agreement. Also check time to time end user experience so that more quality can be maintained. Also check for correct functioning of the application from various geographical locations.

\subsection{Database testing:}

For any project we need to maintain the data in data base. Database applications play an important role in nearly every organization, yet little has been done on testing of database applications. They are becoming increasingly complex and are subject to constant change. They are often designed to be executed concurrently by many clients. Testing of database application hence is of utmost importance to avoid any future errors encountered in the application, since a single fault in database application can result in unrecoverable data loss. Many tools and frameworks for performing testing of database applications has been proposed to populate the test database and generate test cases which checks the correctness of application. They check database applications for consistency constraints and transactions concurrency. In this paper we present a DBGEN- database (test) GENerator, an automated framework for database application testing. In this framework Test Strategies for testing of embedded SQL queries within imperative language are presented. Finally we present strategies for performing efficient regression tests by reducing the resets that may occur while testing database applications. We have also computed the coverage of design various test cases to predict the quality of testing. By this, we reduce the testing time and cost by approximately by $30 \%$, thereby easing the tester to manage his testing activities easily.

So database plays very important role for every organization, so for better results testing of database is required. It is now not only the necessity of project or web application itself but of the organization also to avoid any future problems that can be come in application. As a minute fault in data base can causes data loss that may be uncover able in future. Many tools and frameworks are available for testing of databases or generate test cases to check the applications. In this paper we represent that on which we can apply tests in on database. We have also computed the coverage of design of test cases to maintain the quality of testing. By this, we can decrease the 
time, memory and cost of project to some extent, there by easing the tester to manage their testing phases easily.

Negative and Positive testing: This testing is we give invalid inputs and receive an error that means if database system get any wrong data as input it must give wrong data in response (fig.1). Same way positive test is done that is to give correct input and expect some steps to be completed in accordance with the specification (fig 2).

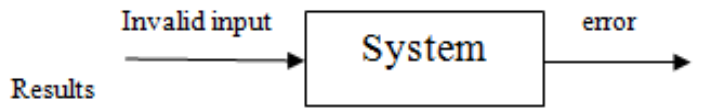

Fig. 1 negative testing

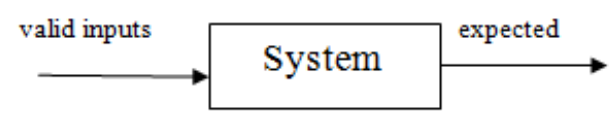

Fig. 2 positive testing

\subsection{Types of testing in database:}

The figure says the areas of testing involved during different databases testing approaches like black-box testing and white box testing

\section{Black Box testing in database:}

In black box testing only testing of output takes places that is not about coding or implementation so in same way black box testing involves interfaces and integration of database, which includes:

- Testing of meta data that is how data is mapped.

- Verifying input data and corresponding output data.

- Checking of data from query languages.

- Various methods used like error guessing, equivalence class partitioning, cause effect graph etc can be used.

For black box testing programmer has good knowledge of designing of database.

\section{White Box Testing in database:}

White box testing basically deals with the internal structure of database. Only testing of output takes a place that is not about coding or implementation so specification details are hidden from user.

- Testing of database triggers, views, etc takes places as they are used for database refactoring.

- Verifying different database functions, views, sql queries etc.

- Checking of database tables, data models, schema etc.

- Checking of database consistency and integrity constraints.

- Various methods are used like condition coverage, decision coverage, statement coverage, cyclometric complexities etc.

For white box testing internal knowledge of database is needed due to this internal bugs can be removed from database. But sometimes in this all SQL statements are not covered.

\section{White Box Database Application Technique:}

It may be noted that generation of test cases is very important in any testing so while creating test cases for database testing, the semantics of SQL queries must be reflected in all test cases. 
So to make this effectively a method is used called as "White Box Database Application Technique".

In this method, SQL queries are covered into GPL statements, followed by traditional white box testing to generate test case that include SQL semantics.[4]

\subsection{Database testing cycle:}

In database life cycle there are various stages like set fixture, test run, verification of output and tear down.

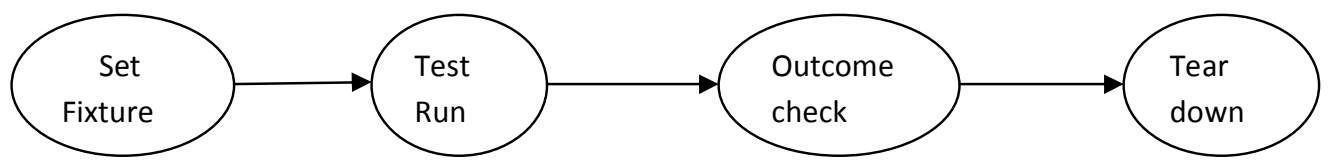

Fig. 3 database testing cycle

In set fixture, is the starting step of database before we start the database testing. Till now test cases have been made so in next step we can test the test cases that are defined for the database. now for this various methods are used $\mathrm{n}$ depending on the results we can decide that test cases should be modified or kept as it is and last is tear down stage that results in either terminating testing or start with other test suite(fig.3).

\section{Some problems in database testing:}

Major problem of database testing are the cost and size of database as set up for database testing is very high in terms of cost and as size is considered then its complex to maintain the whole database. Sometimes extra overhead is occurs when transaction testing takes places.

\subsection{Automated testing:}

Tools available for website testing: There are various tools are available for testing the software at different levels like Jmeter, Grinder, Multi-Mechanize, Selenium, Capybara, Capybara, Pylot, Open STA, Web load, Webrat. There are various tools are available for performing database testing.

AETG: it generates test cases from requirement model. It uses combinatorial design methods to find minimal test case sets to cover all input values.

Data Factory: It is data generator tool and works as a data manager for database testing. Its fast and easy source of data .

Data Generator: It is automated data generation tool which enables you to create test data for assurance of software quality and performance of database which includes database load, stress and endurance of database. It usually generates random test data for purpose of various types of testing like system, integration, module etc.

Test Data Generator: It used to generate data, tables (views, procedures etc) for database testing. 
Datatect: It is powerful software tool for generating varieties of realistic data to ASCII files and RDBMS includes Oracle, Sybase, and SQL Server.

Jenny: It is tool used for carrying out regression testing properly. Always exhaustive testing or condition of endurance testing is creates problems so it covers most of the test cases.

\section{TESTING CRITERIA}

There are some criteria should be decided about when to start and stop the testing.

\subsection{Testing starting criteria}

There is another issue that when to start the testing. So for this, Timing is the major factor that as soon as we get software requirements or baselines we can start testing because incorrect requirements results wrong design and implementation and after implementation has been done it becomes very difficult and also expensive to find the defects and correct them. Every project has some requirements and this has two perspectives; one is from user importance and other is according to user usage, depending olupon these two characteristics a requirement can be generated and a plan or strategy can be made which also means that estimates change accordingly. So objective for starting testing is to trap the requirements related defects as early as possible.

\subsection{Testing completion criteria}

As $100 \%$ testing is not possible and also it's not possible to make the entire defect free software but we need to consider some parameters or factors on the basis of that we can stop the testing process like

- Deadlines as release deadlines, testing deadlines of the project,

- Test cases completed with certain percentage passed .

- Alpha and Beta testing period ends or as we reach optimum level of testing.

\section{DEFECT}

Defect is any flaw in the software system. When we test the website or any web application and there is difference between expected results and the actual results, there is defect. Defects can be classified in to 3 categories: Wrong, Missing and extra (table 1). There are many examples of defects that can come in testing the website or any web application like:

Table 1: Categories of defects

\begin{tabular}{|c|c|}
\hline Category & Examples \\
\hline Wrong & $\begin{array}{l}\text { - User gives wrong/incomplete requirements for developing web } \\
\text { application. Error in coding, in testing, Data entry errors also } \\
\text { Mistakes in error correction or Analyst interprets requirements } \\
\text { incorrectly }\end{array}$ \\
\hline Missing & - Incorrect design specifications or missed out some any specifications. \\
\hline Extra & $\begin{array}{l}\text { - Developer done something extra in web application but client doesn't } \\
\text { require. Poor documentation. }\end{array}$ \\
\hline
\end{tabular}




\subsection{Defect life cycle:}

In defect life cycle there are various defects like new, open, review, rejected, test verified, not a defect etc (fig 4). Defect age or phase age is the important concept in testing that means later we find the defect the more it cost to fix it. Defect spoilage is the concept which works on same concept that how late we find the defects or bugs. When defects are getting fixed during defect life cycle then Retesting and Regression testing is performed. Retesting is testing is performed to check that defect get fixed or not while in regression testing is performed to check that checked tests should not affect the unchecked tests.

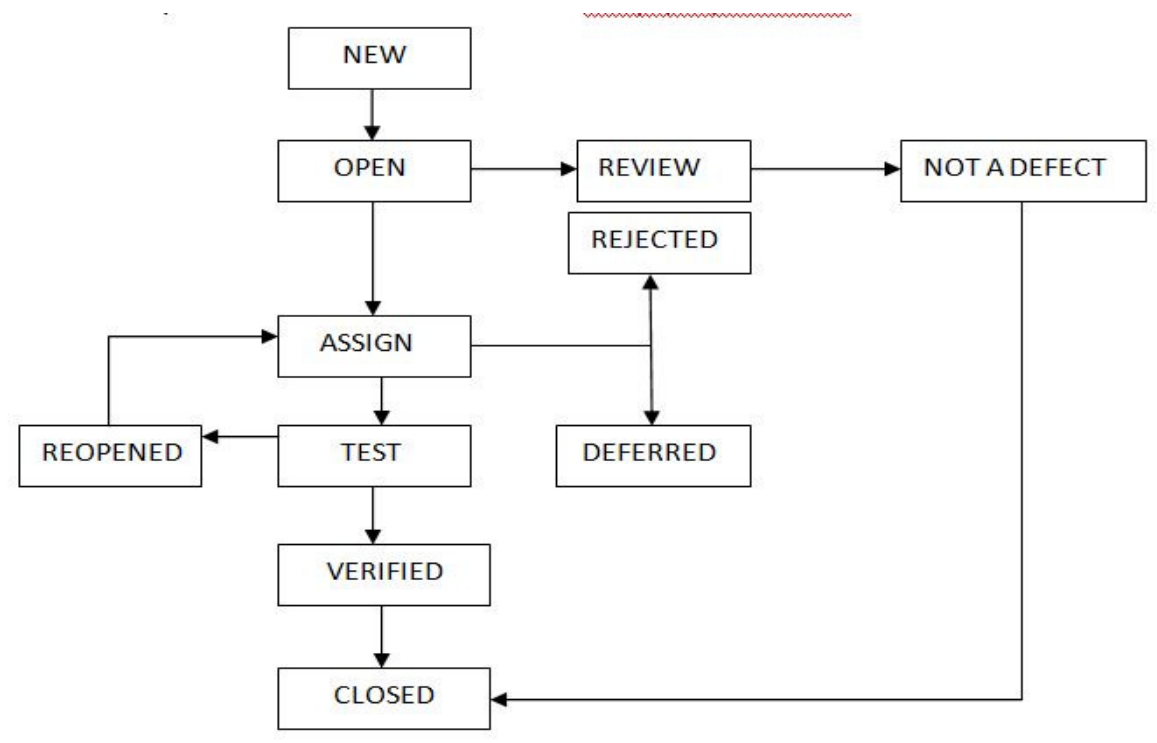

Fig. 4 States of defects

\subsection{Severity of defects:}

Severity shows how bad the bug is and reflects its impact to the product and to the user. This changes from organization to organization or varies from projects to projects (table 2).

Table 2: Severity of defects

\begin{tabular}{|l|l|l|}
\hline Severity & Description & Criteria \\
\hline 1 & Very high & $\begin{array}{l}\text { Inability to install/uninstall the product, product will } \\
\text { not start, product hangs or operating system freezes, } \\
\text { data corruption, product abnormally terminates etc so } \\
\text { they are also called showstopper defects. }\end{array}$ \\
\hline 2 & High & $\begin{array}{l}\text { Function is not working according to specifications, } \\
\text { critical to customer etc that means application can } \\
\text { continue with severe defects. }\end{array}$ \\
\hline 3 & Medium & $\begin{array}{l}\text { Incorrect error message, incorrect data, etc means } \\
\text { application continue with unexpected results. }\end{array}$ \\
\hline 4 & Low & $\begin{array}{l}\text { Spelling, grammar mistakes etc that is defects with } \\
\text { these severities are suggestions given to client to } \\
\text { make application better. }\end{array}$ \\
\hline
\end{tabular}




\subsection{Priority of defects:}

Priority can be decided on the basis of how frequently the defect occurs i.e. probability of occurrence of defect (table 3).

Table 3: Priority of defects

\begin{tabular}{|l|l|l|}
\hline Priority & Description & Criteria \\
\hline 1 & Very high & $\begin{array}{l}\text { Immediate fix, block further } \\
\text { testing }\end{array}$ \\
\hline 2 & High & $\begin{array}{l}\text { Must fix before product is } \\
\text { released }\end{array}$ \\
\hline 3 & Medium & Should fix if time permits \\
\hline 4 & Low & $\begin{array}{l}\text { Would like fix but can be } \\
\text { released as it is }\end{array}$ \\
\hline
\end{tabular}

\section{CONCLUSION \& FUTURE WORK}

Testing is assurance of quality of any kind of software so in order to develop the quality software or web project testing is an essential phase of any development cycle. Testing is widely used now days to help the developers to make the defect free software or any web applications. Although there are so many testing techniques are there but still main important things to do the proper defect management of the defects that occurs. According to the severity and priority of the defects, these can be fixed before deliver product to the client. Defect age and defect spoilage is also important concepts about defect management in web site implementation or web applications as to make the web site error free in optimum level of testing. In this paper, investigation of testing methodologies, and in addition of that focus on test cases design, database testing and defects categories for web based projects are discussed. In near future, the presented literature is utilized to develop a web testing tool.

\section{REFERENCES}

[1] "What Is a Good Test Case"Cem Kaner, J.D., Ph.D. Florida Institute of Technology Department of Computer Sciences kaner@kaner.com STAR East, May 2003

[2] Software testing best practices,Ram Chillarege,IBM Research-Technical report RC 21457

[3] Software Testing Techniques- Shivkumar Hasmukhrai Trivedi [B.com, M.Sc I.T (Information Technology), P.G.D.B.M -Pursuing] Senior System Administrator, S.E.C.C [Socio Economic and Cast Census] Central Govt. Project - Bhavnagar [Gujarat - India], Volume 2, Issue 10, October 2012 ISSN: 2277 128X International Journal of Advanced Research in Computer Science and Software Engineering

[4] Software Testing Research and Software Engineering Education-Thomas J. Ostrand,Elaine J. Weyuker, FoSER 2010, November 7-8, 2010, Santa Fe, New Mexico, USA..Copyright 2010 ACM 978-1-4503-0427-6/10/11 ...\$10.00.

[5] Software Engineering by Roger Pressman

[6] Test Case Prioritization Techniques, Siripong Roongruangsuwan, Jirapun Daengdej, Journal of Theoretical and Applied Information Technology@ 2005 - 2010 JATIT \& LLS. All rights reserved.

[7] Test result reporting by Indira R(white paper of Infosys)

[8] A Study on Software Testing ,H.S. Samra ,Volume 3, Issue 1, January 2013 ISSN: 2277 128X International Journal of Advanced Research in Computer Science and Software Engineering

[9] A parametric approach for security testing of internet application, Arun K Singh,Anand J Iyer, Venkat Seshadri (white paper of Infosys)Enabling Performance Testing with functional testing tool,by Dick Van Driel,Surya Prakash(white paper of Infosys) 
[10] International Journal of Database Theory and Application Vol. 2, No. 3, September 200927 DBGENDatabase (Test) GENerator -An Automated Framework for Database Application Testing 1Askarunisa A., 2Prameela P, and 3Dr. Ramraj N 1,2Thiagarajar College of Engineering, Madurai, Tamilnadu, India

[11] http://sqa.fyicenter.com/FAQ/Testing-Tools/Database_Testing_Tools.html

\section{AUTHORS}

\section{Ms. Bharti Bhattad}

I completed B.E. in computer science from Indore Institute of Science And Technology and pursuing M.tech in software engineering. I also completed diploma in testing from seed InfoTech, Pune. I published some papers on Software Testing. My areas of interests are software testing, Manual testing, test cases design, bug reporting etc.

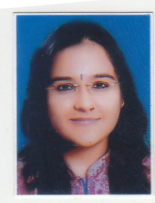

\section{Dr. Abhay Kothari}

He completed Ph.D., MS and B.E..Currently he is working as Professor at Acropolis institute of technology and research. He has 23 years of Working experience. His 18 International and 15 National papers are got Published he get award He get award of IT Excellence by Ministry of Information Technology. 